\title{
Untersuchung eines bei einer Atombombenexplosion entstandenen Teildens
}

\author{
Von Albert Sittkus \\ Max-Planck-Institut für Kernphysik Heidelberg, Außenstelle Freiburg-Schauinsland \\ (Z. Naturforschg. 19 a, 991-999 [1964] ; eingegangen am 22. April 1964)
}

\begin{abstract}
Im radioaktiven Aerosol der atmosphärischen Luft wurde ein „heißes“ Spaltproduktteilchen von $11 \mu \mathrm{m}$ Durchmesser gefunden. Aus Messungen der Zr-Nb-95-Aktivität wurde der Entstehungstag als der 19.12.1962 ( $\pm 1 \mathrm{Tag})$ bestimmt. Gamma-Strahl-Analysen ergaben einen Anfangsgehalt von $11,2 \cdot 10^{8} \mathrm{Zr}-95-, 1,2 \cdot 10^{8} \mathrm{Ba}-140-, 2,4 \cdot 10^{8} \mathrm{Ce}-141-$ und $6,4 \cdot 10^{8} \mathrm{Ce}-144$-Atomen. Mikrosondenmessungen an 3 Bruchstücken zeigten, daß die Hauptmasse des Teilchens von Eisen- und Aluminiumoxyd (je etwa $2 \cdot 10^{13} \mathrm{Fe}$ - und Al-Atome) herrührte. Mit Hilfe der Feuerballmodelle von Mrleter werden Abschätzungen über die Entstehung, Zusammensetzung, Stärke und Art der erzeugenden Explosion durchgeführt. Danach stammt das Teilchen von einer Bombe mit 100-300 kT TNT-Sprengkraft mit Plutonium-239 als spaltbarem Material.
\end{abstract}

Bei Messungen des radioaktiven Aerosols der atmosphärischen Luft auf dem Schauinsland bei Freiburg im Breisgau wurde am 28.12. 1962 ein stark radioaktives Teilchen eingefangen (Anfangsaktivität etwa $10^{-7}$ Curie). Die Partikel wurde auf dem Filter lokalisiert, zusammen mit einem Scheibchen von $2 \mathrm{~mm}$ Durchmesser abgetrennt und eingehend untersucht. Das Ziel war herauszufinden, welche Aufschlüsse über die Bildung dieses Einzelteilchens und allgemein des Spaltproduktaerosols einer Atombombenexplosion gewonnen werden können.

Zwischen Januar und Juni 1963 wurden zahlreiche $\gamma$-Spektren aufgenommen. Abb. 1, Kurve a,

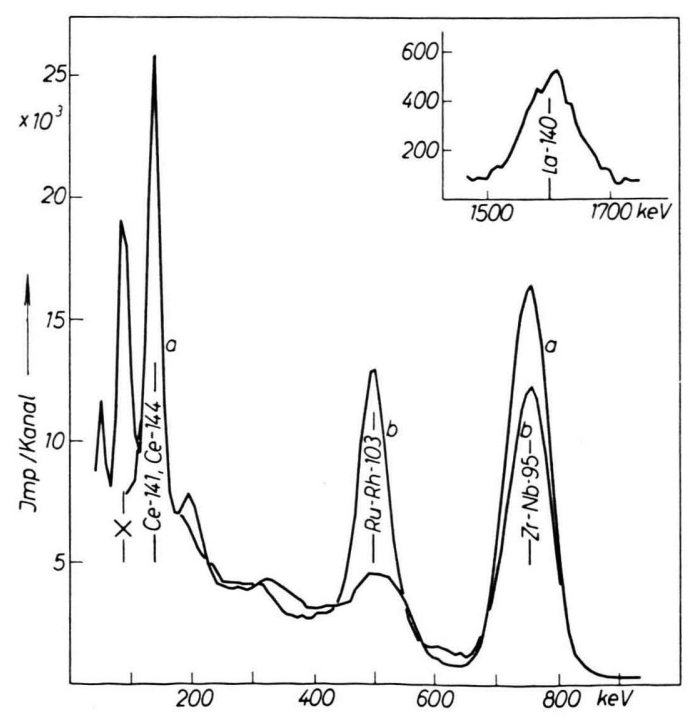

Abb. 1. $\gamma$-Spektren (a) des Teilchens am Sa. 19.1. 1963 und zum Vergleich (b) einer Regenwasserprobe. zeigt ein Beispiel. Im Spektrum dominierte die beim Zerfall von Zr-95 (65 d) und der Tochter Nb-95 (35 d) entstehende $\gamma$-Strahlung (drei nicht aufgelöste $\gamma$-Linien mit 726, 760 und $762 \mathrm{keV})$. Die Linie bei $140 \mathrm{keV}$ rührt von Ce-141 (33 d, $145 \mathrm{keV})$ und Ce-144 (284 d, 134 keV) her. Die markante Linie des Ru-103 (40 d, $495 \mathrm{keV}$ ) und des Paares RuRh-106 (Ru $1 \mathrm{a},-$; Rh $30 \mathrm{~s}, 510 \mathrm{keV})$, welche im $\gamma$-Spektrum von Spaltproduktproben auftritt, die bei der Routineüberwachung der atmosphärischen Luft und der Niederschläge anfallen, Kurve b, ist nicht erkennbar. Nach Abzug des von den anderen Strahlern herrührenden Untergrundes verbleibt im Bereich der $500 \mathrm{keV}$-Linie eine Restintensität, die 5\% der Zr-Nb-95-Aktivität nicht übersteigt. Dieses Ergebnis ist im Einklang mit den Resultaten anderer Messungen an Einzelteilchen ${ }^{1}$ und zeigt, daß bei der Bildung der Teilchen eine starke Fraktionierung des Spaltproduktgemisches eintritt.

Um zu untersuchen, ob die beobachtete spezielle Zusammensetzung der einzeln meßbaren aktiven Partikel auch bei den „heißen“ Teilchen auftritt, die in Kontaktautoradiographien feststellbar sind, deren Aktivität aber für eine Einzelanalyse aus meßtechnischen Gründen nicht ausreicht, wurden nach autoradiographischen Aufnahmen Teilchen kleiner Aktivität isoliert und in Gruppen zusammengefaßt gemessen. In Abb. 2 ist als Beispiel das Ergebnis der Analyse eines Luftfilters wiedergegeben mit dem bei einem Stratosphärenflug ${ }^{2}$ im Dezember 1962 etwa

1 Zum Beispiel Heft 12 der Schriftenreihe „Strahlenschutz“ des Bundesministers für Atomkernenergie und Wasserwirtschaft, Verlag Gersbach u. Sohn GmbH, Braunschweig 1960 oder A. Siтtкus, Naturwiss. 46, 399 [1959]. 


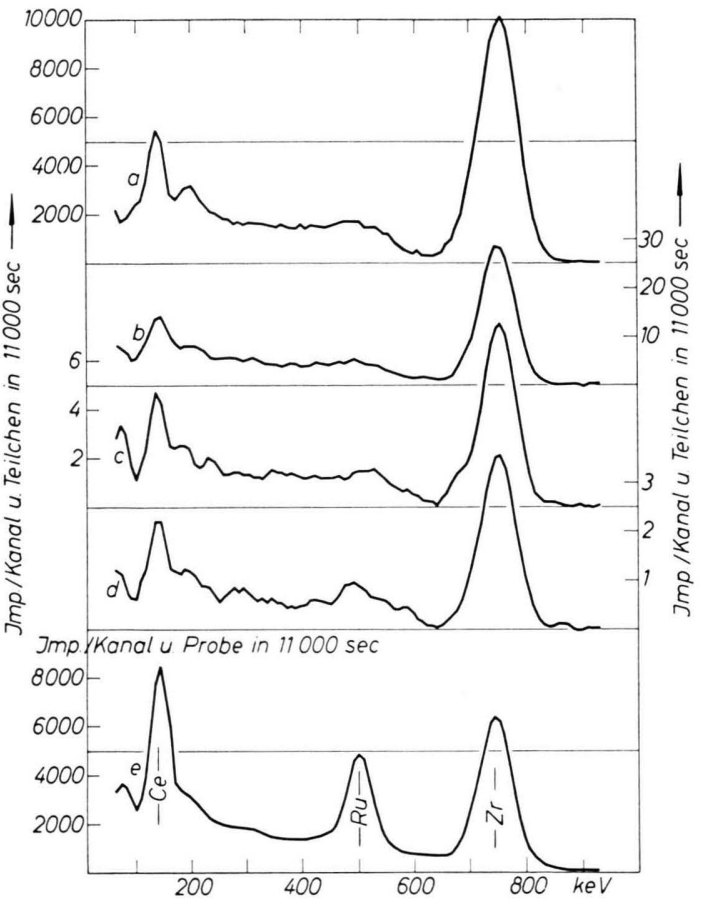

Abb. 2. $\gamma$-Spektren 2-4 Monate alter heißer Teilchen verschiedener Größe. b), c) und d) mittleres Spektrum einer Probe aus 40 bzw. 104 bzw. 167 Teilchen mit etwa 25 bzw. 7 bzw. $3 \cdot 10^{-11}$ Curie/Teilchen, zum Vergleich a) Spektrum des untersuchten Einzelteilchens mit etwa $10^{-7}$ Curie Anfangsaktivität am 10.4.1963 und e) Spektrum des Filterrestes, der Teilchen mit höchstens $1 \cdot 10^{-11}$ Curie/Teilchen enthielt.

$2-5$ Monate altes Spaltproduktgemisch erfaßt wurde. Die Kurven b, c und d zeigen das mittlere $\gamma$-Spektrum von Teilchen mit etwa $25 ; 7$ und $3 \cdot 10^{-11}$ Curie Anfangsaktivität. Zum Vergleich ist in Kurve a das $\gamma$-Spektrum der untersuchten Partikel im Alter von 4 Monaten und in Kurve e das des Filterrestes, der Teilchen mit höchstens $1 \cdot 10^{-11}$ Curie enthielt, dargestellt. In den „heißen“ Teilchen sind die Massenzahlen 103 und 106 beim Einbau stark benachteiligt worden. Sie müssen bevorzugt an Aerosolteilchen angelagert sein, die nur wenige radioaktive Atome enthalten.

Außer den Hauptkomponenten Zr-Nb-95, Ce-141 und Ce-144 wurden in der hier untersuchten Partikel noch zwei kurzlebige Komponenten festgestellt, die zur Aussendung von $\gamma$-Linien bei etwa 80 und $1600 \mathrm{keV}$ führten. Die Untersuchung der zeitlichen

2 Ich danke Herrn Prof. Dr. O. Huber und Herrn Dr. J. Halter, Physikalisches Institut der Universität Freiburg, Schweiz, für ihr Entgegenkommen und ihre Unterstützung bei der Durchführung des Testfluges.
Änderung der $1600 \mathrm{keV}$-Linie ergab, daß sie vom Zerfall des La-140 (40,2 h; 1,597 keV), der Tochter des Ba-140 (12,8 d) stammte. Im Bereich zwischen 100 und $800 \mathrm{keV}$ sind weitere Linien der BaLa-140-Komponente angedeutet. Eine einwandfreie Zuordnung der $80 \mathrm{keV}$-Linie war nicht möglich. Die Halbwertszeit des dazugehörenden Isotopes lag zwischen 2,5 und 7 Tagen.

Nach Abschluß der Meßserie zur Bestimmung der $\gamma$-strahlenden Isotope wurde das heiße Teilchen von dem übrigen Aerosol und der Filterunterlage getrennt. Dabei wurde die $\beta$-Aktivität kontrolliert und sichergestellt, daß die gemessene Aktivität von nur einem Teilchen herrührte. Die mikroskopische Untersuchung zeigte ein kugelförmiges Teilchen mit $11 \mu \mathrm{m}$ Durchmesser und metallisch glänzender Oberfläche. Bei der Übertragung auf eine für weitere Messungen geeignete Unterlage zersprang die Partikel in zahlreiche zum Teil sehr kleine Bruchstücke. Von diesen konnten drei mit etwa 50, 10 und 10\% der ursprünglichen Masse mit einer Mikrosonde System Cameca auf ihre Elementzusammensetzung untersucht werden ${ }^{3}$. Der Hauptbestandteil der Bruchstücke war Eisen, als nächst häufiges Element wurde Aluminium gefunden in Übereinstimmung mit ähnlichen Messungen von Rajewsky ${ }^{4}$. Mit Anteilen unter 1 Gew.-Proz. wurden außerdem noch Titan, Vanadium, Chrom, Mangan, Nickel und Kupfer festgestellt. Bei Zirkon, Niob und Uran wurden keine die Erkennbarkeitsgrenze dieser Elemente $(1,0 ; 1,8$; $0,9 \%)$ übersteigenden Gehalte festgestellt. Auffällig war die starke Schwankung des Aluminiumgehaltes der Trümmer. Bezogen auf Eisen variiert der Gewichtsanteil von Aluminium zwischen 5 und $70 \%$. Das aufgefangene Teilchen war inhomogen.

Zur Abschätzung der Kondensationsvorgänge im Feuerball einer Atombombenexplosion ist die Kenntnis des Zustandes unmittelbar nach dem Ablauf der Kondensationsvorgänge notwendig. Für die zum Zeitpunkt der Messung noch vorhandenen radioaktiven Isotope ist die Bestimmung aus dem Alter der Partikel möglich. Bei dem untersuchten Einzelteilchen mit nur wenigen gut meßbaren Isotopenarten wurde es aus dem Vergleich von $\mathrm{Zr}-95$ und Nb-95 bestimmt (Zirkon-Niob-Alter). Niob-95 entsteht bei der Spaltung nicht direkt, sondern ist das

3 A. Sittkus u. L. Lehmann, Naturwiss., im Druck.

4 M. F. Rajewsky, Nature, Lond. 199, 162 [1963]. 
letzte instabile Glied der Reihe

$$
\begin{aligned}
& \mathrm{Kr}(1 \mathrm{~s}) \rightarrow \mathrm{Rb}(1 \mathrm{~s}) \rightarrow \mathrm{Sr}(42 \mathrm{~s}) \\
& \text { 4l,1 } 179 \quad 193
\end{aligned}
$$

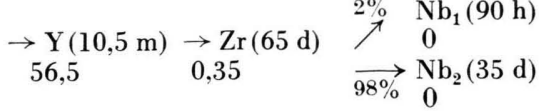

(Neben dem Elementsymbol steht in Klammern die Halbwertszeit, darunter die Spaltausbeute bei 10000 Spaltungen. Die als Beispiel gewählte Reihe entsteht bei der Spaltung von U-238 mit Neutronen von $14 \mathrm{MeV}^{5}$. Für andere Isotope und Neutronenenergien ergeben sich geringe Änderungen der Spaltausbeute.) Nach dem Ablauf des Explosionsvorganges (je nach dem Bombenkaliber bis zu einigen Minuten ${ }^{6}$ ) enthält ein kondensiertes Teilchen vom Ablauf der Explosion abhängige unbekannte Menge Rubidium, Strontium, Yttrium und Zirkon, aber nur sehr wenig Niob. Rb-, Sr- und Y-95 zerfallen schnell zu Zr-95 und der Zerfall zu Nb-95 beginnt mit vernachlässigbarer Niob-Aktivität zu einem Zeitpunkt, nach dem nur der radioaktive Zerfall die Zirkon- und Niob-Anteile ändert ${ }^{7}$. Kernreaktionen in nicht spaltbarem Material werden kaum zur unmittelbaren Bildung von Nb-95 führen. Neutronenumwandlungen geben $\mathrm{Zr}-95\left(\mathrm{Zr}^{94}(\mathrm{n}, \gamma) \mathrm{Zr}^{95} \rightarrow \mathrm{Nb}^{95}\right)$. In Fusionsbomben mögliche Reaktionen von geladenen Teilchen, z. B. $\mathrm{Mo}^{97}(\mathrm{~d}, \alpha) \mathrm{Nb}^{95}$ oder $\mathrm{Zr}^{94}(\mathrm{~d}, \mathrm{n}) \mathrm{Nb}^{95}$ können wohl wegen der speziell notwendigen seltenen Elemente und der zu erwartenden kleinen Ausbeuten unberücksichtigt bleiben. Für die Altersbestimmung wurde daher angenommen, daß unmittelbar nach der Teilchensynthese noch kein Nb-95 vorhanden war. Dann gestattet die Messung der Zeitabhängigkeit der Zr- und Nb-Aktivität die Festlegung des Zeitpunktes der Zirkonbildung und damit der Explosion ${ }^{8}$. Der Zerfall des Zr-95 führt in 98\% der Fälle zur Aussendung eines $\gamma$-Quants von 726 (55\%) bzw. $760 \mathrm{keV} \mathrm{(43 \% ),} \mathrm{der} \mathrm{des} \mathrm{Nb-95} \mathrm{mit}$ 99\% Häufigkeit zu einem Quant von $762 \mathrm{keV}$. Unter Vernachlässigung der kleinen Unterschiede der Ansprechempfindlichkeit des Detektors und der $\gamma$-Strahlausbeuten beim Zerfall ist die „Summenlinie“ bei $750 \mathrm{keV}$ eine der Summe der Zirkon- und Niob-95-

5 P. J. Dolan, Calculated Abundances and Activities of the Products of High Energy Neutron Fission of Uranium-238, Dasa 525, Mai 1959, Headquarters Defense Atomic Support Agency, Washington 25, D.C.

6 C. F. Mrleer, Fallout and Radiological Countermeasures, Vol. 1, Stanford Research Institute, Manlo Park, Califor-

7 Nach dem Ende der Explosion unterliegt das Teilchen dem Einfluß der Atmosphäre. Diese kann Änderungen der
Aktivität proportionale Größe. Aus dem Verlauf der Aktivität ergab sich das Alter der Partikel bei der Abscheidung am 28.12. 1962 zu 9( \pm 1$)$ Tage. Dieses Teilchen wurde in der Zeit vom 18. bis 20. 12 . 1962 gebildet (nach den Zusammenstellungen der A E C wahrscheinlich bei einer von drei Explosionen im nördlichen Eismeeer, zwei am 18. 12. 1962 von mittlerem Kaliber; eine am 20.12.1962 mit geringer Energie).

Nach dem Abklingen der störenden Ba-La-140Komponente wurden die Zerfallsraten von Ce-141 und Ce-144 einzeln bestimmt. Schließlich wurden sämtliche Werte auf den Explosionstag (19.12. 1962) reduziert und nach Eichung der Apparatur mit annähernd punktförmigen Reinpräparaten von Zr-Nb-95, Ba-La-140, Ce-141 und Ce-Pr-144 die Anfangsintensitäten und Atomzahlen errechnet. Das Teilchen enthielt ursprünglich $(11,2 \pm 0,8) \cdot 10^{8} \mathrm{Zr}$ 95-, $(1,21 \pm 0,08) \cdot 10^{8}$ Ba-140-, $(2,36 \pm 0,17) \cdot 10^{8}$ Ce-141 und $(6,38 \pm 0,45) \cdot 10^{8} \mathrm{Ce}-144$-Atome. Die Abschätzung der Zahl der Eisen- und Aluminiumatome ergibt mit der Annahme, daß diese Elemente als Oxyde vorliegen, je etwa $2 \cdot 10^{13} \mathrm{Fe}$ - und AlAtome. Wegen der Unsicherheiten der Mikrosondenmessungen ${ }^{3}$ und der Unmöglichkeit der einwandfreien Ermittlung der Zusammensetzung der ursprünglichen Partikel aus den Werten der drei untersuchten Bruchstücke ist die Genauigkeit dieser Bestimmung gering. In Tab. 1 sind relative Ausbeuten verschiedener Massenzahlen bei der Uran- und Plutoniumspaltung mit den gemessenen Werten der untersuchten Partikel zusammengestellt. Die Aufstel-

\begin{tabular}{|c|c|c|c|c|}
\hline Massenzahl & 95 & 140 & 141 & 144 \\
\hline $\mathrm{U}^{235}$ mit Spaltneutronen 6 & 100 & 102 & 100 & 81 \\
\hline Spaltneutronen 6 & 100 & 100 & 100 & 86 \\
\hline $\begin{array}{l}14 \mathrm{MeV} \text {-Neutronen }{ }^{1} \\
\mathrm{Pu}^{239} \text { mit }\end{array}$ & 100 & 98 & 92 & 70 \\
\hline Spaltneutronen ${ }^{6}$ & 100 & 89 & 84 & 86 \\
\hline im Teilchen gefunden & 100 & 11 & 21 & 57 \\
\hline
\end{tabular}
lung zeigt die Bevorzugung des Zirkon und die

Tab. 1. Auf die Masse 95 bezogene Ausbeuten verschiedener Massenzahlen bei der Uran- und Plutoniumspaltung im Vergleich zu den im heißen Teilchen gemessenen Werten.

Struktur und Zusammensetzung hervorrufen. Doch kann wohl angenommen werden, daß der Gehalt der hier untersuchten Elemente unverändert bleibt.

${ }^{8}$ Die günstigere Messung des Verhältnisses von $\mathrm{Zr}-95 \mathrm{zu}$ Nb-95 hätte eine chemische Aufarbeitung erfordert. Dadurch wären andere Untersuchungen des Teilchens erheblich erschwert worden. 
starke Veränderung des Ce-141/Ce-144-Verhältnisses im Teilchen.

Durch Verwertung quantitativer Angaben von Miller ${ }^{6}$, der die mechanisch großen Partikel des lokalen Fallout einer Bodenexplosion analysierte, wurde versucht, die Vorgänge, die zur Bildung des untersuchten Einzelteilchens führten, zu verstehen. Als Modell zur Abschätzung des Kondensationsverhaltens verschiedener Elemente wurde eine Luftexplosion (der homogen angenommene Feuerball berührte niemals den Erdboden) gewählt ${ }^{9}$. Die Zeitabhängigkeit der Temperatur wird beschrieben durch $T=5,95 \cdot 10^{3} W^{-0,020} e^{-0,0389 t / t_{2}{ }^{\circ}} \mathrm{K}, \quad t / t_{2} \geqq 10$, $t_{2}=0,050 \cdot W^{0,413} \sec (W$ in Kilotonnen TNT).
Der Faktor $W^{-0,020}$ ist nur wenig energieabhängig. Er wurde durch den Wert 0,9 ersetzt. Dann beschreibt die Gleichung

$$
T=5,36 \cdot 10^{3} e^{-0,0398 t / t_{2}{ }^{\circ} \mathrm{K}, \quad t / t_{2} \geqq 10,}
$$

den Temperaturverlauf für Explosionsenergien zwischen 1 und $10^{5} \mathrm{kT}$ mit einer Genauigkeit von $\pm 10 \%$. Aus der Temperaturkurve wurde für Eisen, Aluminium und verschiedene Spaltproduktelemente die Zeitabhängigkeit des Sättigungsdampfdruckes der Oxyde berechnet und in den Kurven $1-11$ der Abb. 3 dargestellt. Dabei wurden die Verdampfungsreaktionen ausgewählt, die zur höchsten Zahl von Atomen des betreffenden Elementes in der Gasphase führten ${ }^{10}$. Soweit der Sauerstoffpartialdruck eine

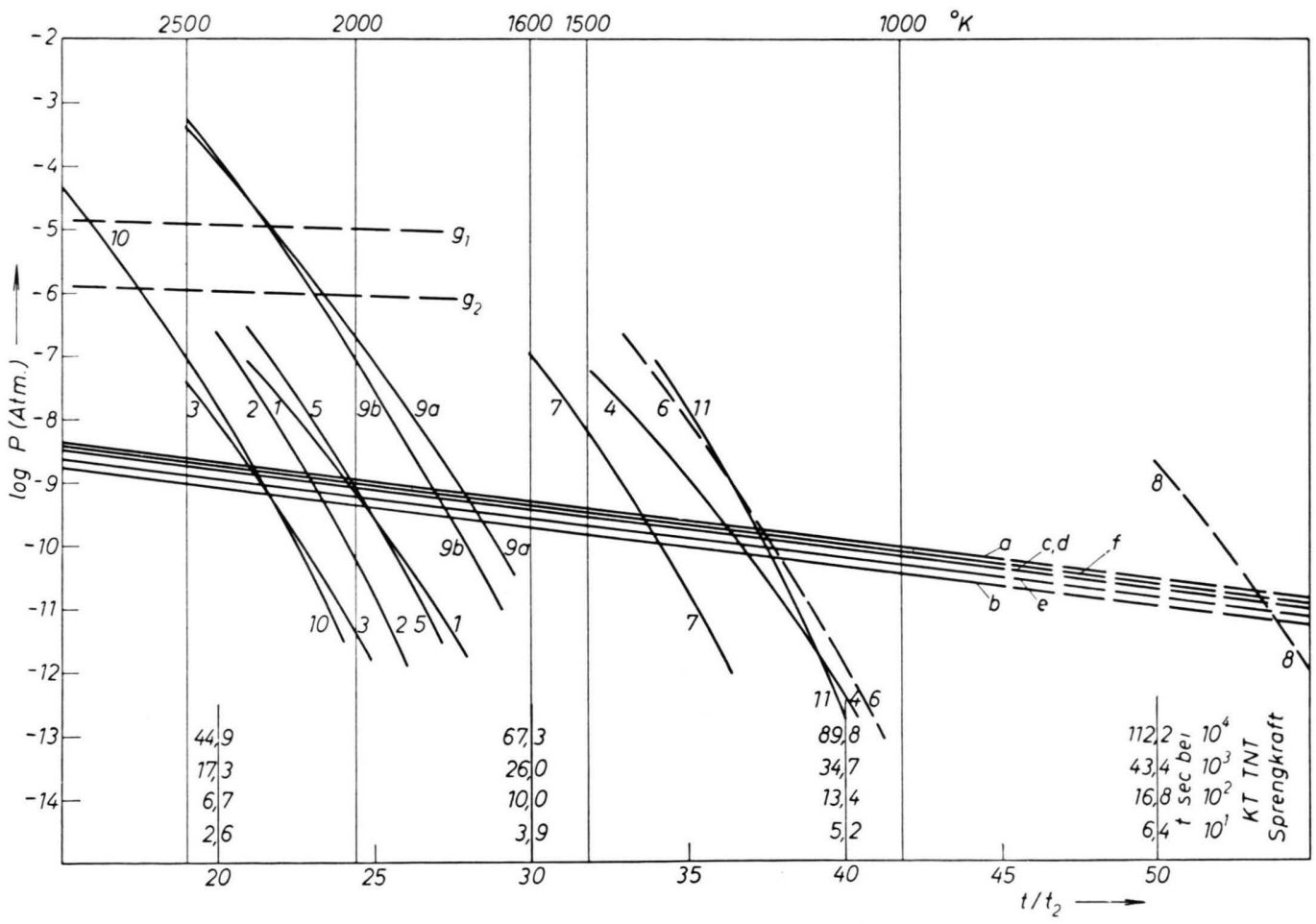

Abb. 3. Verlauf des Partialdruckes und des Sättigungsdampfdruckes verschiedener Elemente, die im Feuerball einer Plutonium-239-Atombombenexplosion auftreten.

a) Partialdruck von $\mathrm{Sr}$; b) von $\mathrm{Y}$; c) von $\mathrm{Zr}$; d) von $\mathrm{Ba}$; 7) von $\mathrm{Nb}\left[\frac{1}{2} \mathrm{Nb}_{2} \mathrm{O}_{5}(\mathrm{c}, \mathrm{l}) \rightarrow \mathrm{NbO}_{2}(\mathrm{~g})+\frac{1}{4} \mathrm{O}_{2}(\mathrm{~g})\right]$

e) von La und f) von Ce.

8) von $\mathrm{Cs}\left[\frac{1}{2} \mathrm{Cs}_{2} \mathrm{O}(\mathrm{l}) \rightarrow \mathrm{Cs}(\mathrm{g})+\frac{1}{4} \mathrm{O}_{2}(\mathrm{~g})\right]$

1) Sättigungsdampfdruck von $\mathrm{Sr}[$ Reaktion $\mathrm{SrO}(\mathrm{c}) \rightarrow \mathrm{SrO}(\mathrm{g})]$

9a) von $\mathrm{Fe}\left[1,056 \mathrm{Fe}_{0,947} \mathrm{O}(\mathrm{c}, \mathrm{l}) \rightarrow \mathrm{Fe}(\mathrm{g})+0,528 \mathrm{O}_{2}(\mathrm{~g})\right]$

2) von $\mathrm{Y}\left[\frac{1}{2} \mathrm{Y}_{2} \mathrm{O}_{3}(\mathrm{c}) \leftarrow \mathrm{YO}(\mathrm{g})+\frac{1}{4} \mathrm{O}_{2}(\mathrm{~g})\right]$

3) von $\mathrm{Zr}\left[\mathrm{ZrO}_{2}(\mathrm{c}) \rightarrow \mathrm{ZrO}_{2}(\mathrm{~g})\right]$

4 von $\mathrm{Ba}[\mathrm{BaO}(\mathrm{c}, \mathrm{l}) \rightarrow \mathrm{BaO}(\mathrm{g})]$

5) von $\mathrm{La}\left[\frac{1}{2} \mathrm{La}_{2} \mathrm{O}_{3}(\mathrm{c}) \rightarrow \mathrm{LaO}(\mathrm{g})+\frac{1}{4} \mathrm{O}_{2}(\mathrm{~g})\right]$

6) von $\mathrm{Ce}\left[\mathrm{CeO}_{2}(\mathrm{c}) \rightarrow \mathrm{CeO}_{2}(\mathrm{~g})\right]$

$9 \mathrm{~b})$ von $\mathrm{Fe}\left[\frac{1}{3} \mathrm{Fe}_{3} \mathrm{O}_{4}(\mathrm{c}, \mathrm{l}) \rightarrow \mathrm{Fe}(\mathrm{g})+\frac{2}{3} \mathrm{O}_{2}(\mathrm{~g})\right]$

10) von $\mathrm{Al}\left[\frac{1}{2} \mathrm{Al}_{2} \mathrm{O}_{3}(\mathrm{c}, 1) \rightarrow \mathrm{AlO}(\mathrm{g})+\frac{1}{4} \mathrm{O}_{2}(\mathrm{~g})\right]$

(c $=$ kristallin, $\mathrm{l}=$ flüssig, $\mathrm{g}=$ gasförmig $)$

$\mathrm{g}_{1}$ ) Partialdruck von $\mathrm{Fe}$ und $\mathrm{Al}, 3,3 \cdot 10^{13}$ Atome $/ \mathrm{cm}^{3}$

$\mathrm{g}_{2}$ ) Partialdruck von $\mathrm{Fe}$ und $\mathrm{Al}, 3,3 \cdot 10^{12}$ Atome $/ \mathrm{cm}^{3}$

9 Wenn nicht anders vermerkt, sind für die folgenden Uberlegungen notwendige Werte den Zusammenstellungen von Miller ${ }^{6}$ entnommen.

10 Die Beiträge anderer Verdampfungsreaktionen wurden vernachlässigt, da sie bei den untersuchten Elementen nur geringe Änderungen der Sättigungsdampfdruckkurven bewirkten. 
Rolle spielt, wurde er mit 0,1 Atm. (entsprechend einer Explosion in etwa $5000 \mathrm{~m}$ Höhe) eingesetzt. Die zeitliche Änderung des Radius des Feuerballes der Luftexplosion ist gegeben durch

$R=4,41 \cdot 10^{3} W^{0,333} e^{0,035 t / t_{2}} \mathrm{~cm}, 10 \leqq t / t_{2} \leqq 45$.

Daraus folgt:

$$
V / W=3,59 \cdot 10^{11} e^{0,105 t / t_{2}} \mathrm{~cm}^{3} .
$$

Bei Spaltbomben ist zur Erzeugung der Energie von $1 \mathrm{kT}$ TNT die Spaltung von $1,4 \cdot 10^{23}$ Uran- oder Plutoniumatomen nötig. Dabei entstehen $2,8 \cdot 10^{23}$ Spaltproduktatome. Dann ergibt sich für reine Spaltbomben die Spaltproduktdichte zu

$$
D=7,8 \cdot 10^{11} e^{-0,105 t / t_{2} \text { Spaltatome } / \mathrm{cm}^{3} .}
$$

Für die Elemente Sr, Y, Zr, Ba, La und Ce wurde nach den Angaben von Dolan 5, 11 die Zahl der zu verschiedenen Zeiten nach der Spaltung vorhandenen Atome bestimmt. (Abb. 4 a zeigt als Beispiel den Verlauf für die Elemente Ba, La und Ce.) Zwischen 0 und $180 \mathrm{sec}$ schwankt sie für ein Element um etwa den Faktor 2. Als Näherung wurde der Wert bei 36 sec gewählt und mit (6) der Dichteverlauf der einzelnen Elemente ermittelt. Durch Hinzunahme der Temperaturgleichung (3) und Anwendung des idealen Gasgesetzes wurden aus dem Dichteverlauf der Partialdruckverlauf berechnet und in Abb. 3 Kurven a $-\mathrm{f}$ eingezeichnet.

Aus der Abbildung lassen sich die Zeitpunkte des Beginns der Abscheidung der einzelnen Elemente entnehmen. Die Darstellung zeigt, daß die Reihenfolge des Kondensationsbeginnes durch Änderungen der Partialdrucke nur wenig beeinflußt wird (dies rechtfertigt die oben gemachten Näherungen). Das Modell gestattet daher Abschätzungen des Kondensationsablaufes auch, wenn anderes spaltbares Material verwendet wird (Verschiebung der Partialdruckkurven gegeneinander), wenn neben der Spaltenergie andere Energiequellen (thermonukleare Reaktionen) Beiträge zur Explosionsstärke liefern (gleichmäßige Verschiebung der Partialdruckkurven zu kleineren Werten) oder wenn die Temperatur-

11 Die angegebenen Spaltausbeuten für die verschiedenen chemischen Elemente einer Massenzahl gelten für die Spaltung von U-238 mit 14 MeV-Neutronen. Da für $\mathrm{Pu}-239$ keine detaillierten Angaben verfügbar waren, wurde die Häufigkeit des Auftretens der Elemente einer Massenzahl bei der Pu-239-Spaltung genähert bestimmt durch Multiplikation der Dolanschen Werte mit dem Verhältnis der Massenzahlausbeuten bei Pu-239 und U-238. abnahme und die Radiuszunahme des Feuerballs nach einem anderen Zeitgesetz abläuft, z. B.

$$
\begin{aligned}
& T \sim W^{0,010} e^{-0,0333 t / t_{2}}, \\
& R \sim W^{0,333} e^{0,0104 t / t_{2}}
\end{aligned}
$$$$
\text { und } \quad t_{2}=0,061 W^{0,373}
$$

für eine Explosion mit Bodenberührung nach MILLER ${ }^{6}$. Änderungen der Sättigungsdampfdruckkurven durch Variation des Sauerstoffpartialdruckes zwischen etwa 0,2 und $0,02 \mathrm{Atm}$. sind ebenfalls weitgehend unwirksam. Aus der Darstellung ergibt sich daher für Atombombenexplosionen in der unteren Atmosphäre folgendes allgemeine Bild des Ablaufes der Spaltproduktkondensation. Zuerst überschreitet der Partialdruck von Zirkonoxyd den Sättigungsdampfdruck und Zirkonoxyd kondensiert bei etwa $2200{ }^{\circ} \mathrm{K}$. Kurz oberhalb $2000{ }^{\circ} \mathrm{K}$ beginnt die Abscheidung von Yttrium-, Strontium- und Lanthanoxyd. Danach erfolgt zwischen 1500 und $1000{ }^{\circ} \mathrm{K}$ die Ausscheidung der Oxyde von Niob, Barium, Zinn und Cer, und bei Temperaturen um $500{ }^{\circ} \mathrm{K}$, wo die Gültigkeit des verwendeten Modelles sehr fraglich wird, von Cäsium ${ }^{12}$.

Die Abschätzung der Kondensationszeitpunkte für in den Feuerball einbezogenes Material der Bombenstruktur und der Explosionsortumgebung ist schwierig. Die Hauptmasse des untersuchten Teilchens besteht aus Eisen und Aluminium. Daher kann angenommen werden, daß die Partialdampfdrucke der Oxyde dieser Elemente anfänglich wesentlich höher waren als die der Spaltproduktelemente. Dann wird die Kondensation des Aluminiumoxyd noch vor der des Zirkonoxyd erfolgen und Aluminiumoxyd, das bei $2290{ }^{\circ} \mathrm{K}$ erstarrt, kann das feste Trägeraerosol bilden, an das sich Eisenoxyd und die Oxyde von $\mathrm{Zr}$, Y, Sr und La anlagern. Die Kugelgestalt des Teilchens und seine glänzende Oberfläche deuten darauf hin, daß die Kondensation zu flüssigem Eisenoxyd oberhalb von $1650{ }^{\circ} \mathrm{K}$ (Schmelzpunkt von $\mathrm{Fe}_{2} \mathrm{O}_{3} 1650{ }^{\circ} \mathrm{K}$, von $\mathrm{Fe}_{3} \mathrm{O}_{4} 1870{ }^{\circ} \mathrm{K}$ ) erfolgte. In der flüssigen Eisenschmelze können sich die kondensierten Spaltelemente Zr, Y, Sr und La gleich-

12 Grobe Abschätzungen für andere Spaltproduktelemente aus den Massenzahlbereichen 89-106 und 129-149 zeigen, daß Ruthenium mit $\mathrm{Ba}$ und $\mathrm{Ce}$, Antimon, Molybdän, Rubidium, Selen, Technetium und Tellur mit Cs ausgeschieden werden. 


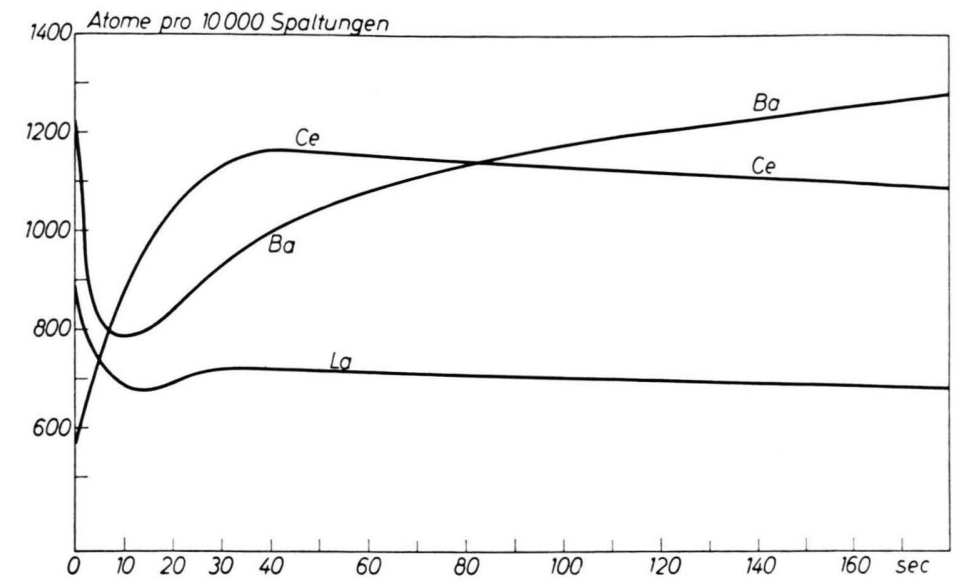

Abb. 4 a. Zeitabhängigkeit der Konzentration der Elemente Barium, Lanthan und Cer bei einer Plutonium-239-Explosion.

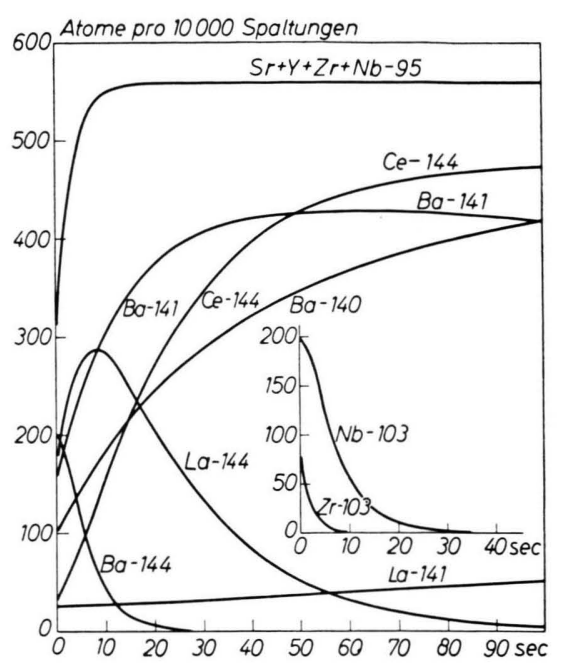

Abb. 4 b. Zeitabhängigkeit der Konzentration verschiedener radioaktiver Isotope bei einer Plutonium-239-Explosion. mäßig verteilen, der oder die darin enthaltenen Aluminiumoxydkerne haben sich, wie die Analyse der Bruchstücke (s. oben) zeigt, höchstens teilweise gelöst, so daß die Partikel inhomogen wurde. Die Kondensation der übrigen Spaltelemente erfolgt erst unterhalb $1650^{\circ} \mathrm{K}$. Diese Elemente werden an der Oberfläche des erstarrten Kondensationskernes angelagert ${ }^{13}$. Infolge der Expansion des Feuerballes vermindert sich die Spaltproduktdichte. Dadurch sinkt die Wahrscheinlichkeit der Abscheidung an den primären Kondensationskernen im Laufe der Zeit ab. Von Oxyden, die erst später kondensieren, wird nur ein Bruchteil der zur Verfügung stehenden Menge an die in einem früheren Stadium gebildeten Aerosolteilchen angelagert, und zwar um so weniger, je später nach der Bildung des Kernes der Partialdruck den Sättigungsdampfdruck der betreffenden Komponente erreicht. Dieses Bild wird durch die experimentellen Beobachtungen belegt, so zeigt z. B. Tab. 1, daß die Masse 140, deren Vertreter Xe, Cs und $\mathrm{Ba}$ erst nach mehreren Elementen der Massenzahl 95 (Sr, Y, Zr) kondensieren, im untersuchten Teilchen stark benachteiligt wurde. Wenn die bei Beginn der Kondensation gebildeten Partikel im Laufe der Feuerballentwicklung nicht alle „anlage-

13 Für die in der Bombenstruktur zu erwartenden Stoffe und auch dann, wenn bei einer Explosion mit Bodenberührung erhebliche Mengen von Silikaten in den Feuerball gelangen, ist zu erwarten, daß $\mathrm{Zr}, \mathrm{Y}, \mathrm{Sr}$ und La meist an flüssige Kerne abscheiden. Dagegen werden die übrigen Spaltelemente überwiegend an erstarrte Kerne kondensieren. rungsbereiten " Atome aufnehmen, so ist zu erwarten, daß laufend weitere Aerosolteilchen mit abweichender Zusammensetzung neu gebildet werden. Soweit bekannt geworden, sind im weltweiten Fallout (dem Teil des Explosionsschwaden, der erst nach einigen Tagen am Boden abgeschieden wird) keine aktiven Teilchen mit mehr als $10^{-11}$ Curie/Teilchen (s. oben) gefunden, bei denen bei vergleichbarem Teilchenalter z. B. Zr-Nb-95 stark vermindert gegenüber Ba-La-140, Ce-141 und Ce-Pr-144 war. Daraus kann geschlossen werden, daß nach der ersten Kondensationsphase, die gekennzeichnet ist durch die Ausscheidung der an Masse überwiegenden Teile der Bombenstruktur und der Explosionsortumgebung, die Kondensations- und Koagulationsbedingungen in der nun sehr verdünnten Gasphase des Feuerballes so ungünstig wurden ${ }^{14,15}$, daß das später gebildete aktive Aerosol aus meßtechnischen Gründen der Einzelteilchenanalyse nicht zugänglich war. Das skizzierte Kondensationsschema gestattet ohne genaue Kenntnis der Entwicklung des Feuerballes und der Kondensations- und Koagulationsvorgänge bei der Aerosolbildung nur grobe Aussagen über die in heiße Teilchen eingebauten Mengen der im Spaltproduktgemisch vorhandenen radio-

14 Zum Beispiel N. A. Furs, The Mechanics of Aerosols, aus dem Russischen übersetzt von E. LAchowics, U.S. Department of Commerce, Office of Technical Services, Washington 25, D.C.

15 K. Stewart, Trans. Faraday Soc. 52, 161 [1956]. 


$$
\begin{aligned}
& \begin{array}{rl}
89 & \mathrm{Se}(2 \mathrm{~s}) \\
260 & 48,5
\end{array} \rightarrow \mathrm{Br}(4,51 \mathrm{~s}) \stackrel{88.2 \%}{\rightarrow} \begin{array}{l}
\mathrm{Kr}(3,18 \mathrm{~m}) \\
83,2
\end{array} \rightarrow \begin{array}{l}
\mathrm{Rb}(15,4 \mathrm{~m}) \\
14,3
\end{array} \rightarrow \begin{array}{l}
\mathrm{Sr}(50,5 \mathrm{~d}) \\
0,029
\end{array} \\
& \begin{aligned}
90 \\
300 \\
23,5
\end{aligned} \rightarrow \begin{array}{l}
\mathrm{Br}(1,4 \mathrm{~s}) \\
105
\end{array} \rightarrow \begin{array}{l}
\mathrm{Kr}(33 \mathrm{~s}) \\
129
\end{array} \rightarrow \begin{array}{l}
\mathrm{Rb}(2,7 \mathrm{~m}) \\
42,0
\end{array} \rightarrow \begin{array}{l}
\mathrm{Sr}(27,7 \mathrm{y}) \\
0,48
\end{array} \rightarrow \begin{array}{l}
\mathrm{Y}(64,2 \mathrm{~h}) \\
0
\end{array}
\end{aligned}
$$

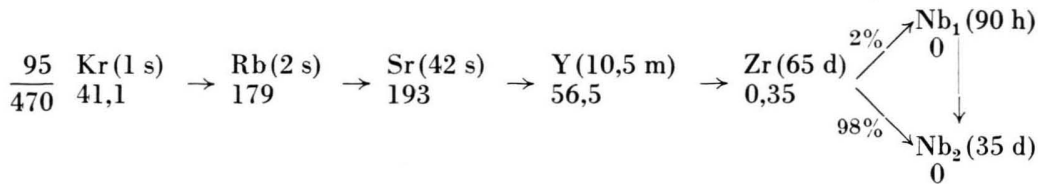

$$
\begin{aligned}
& \begin{array}{rl}
99 & \mathrm{Y}(1,5 \mathrm{~s}) \\
570 & 217
\end{array} \rightarrow \begin{array}{l}
\mathrm{Zr}(30 \mathrm{~s}) \\
254
\end{array} \rightarrow \begin{array}{l}
\mathrm{Nb}(3,8 \mathrm{~m}) \\
97,0
\end{array} \rightarrow \begin{array}{l}
\mathrm{Mo}(66 \mathrm{~h}) \\
2,0
\end{array} \stackrel{13 \%}{\rightarrow} \underset{0}{\mathrm{Tc}_{1}(6,04 \mathrm{~h})} \\
& \begin{array}{l}
103 \mathrm{Zr}(1,5 \mathrm{~s}) \\
450 \\
85,8
\end{array} \rightarrow \begin{array}{l}
\mathrm{Nb}(4 \mathrm{~s}) \\
203
\end{array} \rightarrow \begin{array}{l}
\mathrm{Mo}(25 \mathrm{~s}) \\
140
\end{array} \rightarrow \begin{array}{l}
\mathrm{Tc}(1,2 \mathrm{~m}) \\
21,1
\end{array} \rightarrow \begin{array}{l}
\mathrm{Ru}(39,8 \mathrm{~d}) \\
0,037
\end{array} \rightarrow \begin{array}{l}
\mathrm{Rh}_{1}(57 \mathrm{~m}) \\
0
\end{array} \\
& \begin{array}{l}
106 \\
270 \\
41,7
\end{array} \rightarrow \begin{array}{l}
\mathrm{Mo}(4 \mathrm{~s}) \\
119
\end{array} \rightarrow \begin{array}{l}
\mathrm{Tc}(9 \mathrm{~s}) \\
91,6
\end{array} \rightarrow \begin{array}{l}
\mathrm{Ru}(1 \mathrm{y}) \\
17,6
\end{array} \rightarrow \begin{array}{l}
\mathrm{Rh}(30 \mathrm{~s}) \\
0,038
\end{array}
\end{aligned}
$$

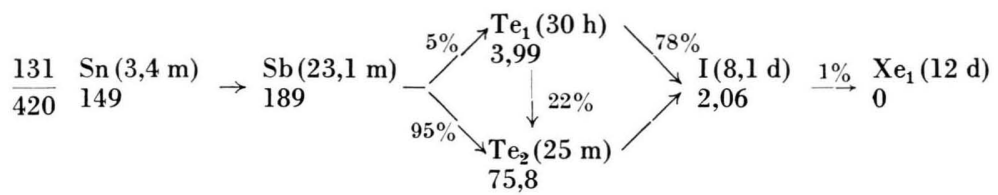

$$
\begin{aligned}
& \begin{array}{ll}
137 \\
540 \\
30,4
\end{array} \rightarrow \begin{array}{l}
\mathrm{Te}(3 \mathrm{~s}(22 \mathrm{~s}) \\
184
\end{array} \stackrel{93,2 \%}{\rightarrow} \underset{238}{\mathrm{Xe}(3,9 \mathrm{~m})} \rightarrow \underset{86,4}{\mathrm{Cs}(26,6 \mathrm{y})} \stackrel{92}{\rightarrow} \underset{1,2}{\mathrm{Ba}_{1}(2,60 \mathrm{~m})}
\end{aligned}
$$

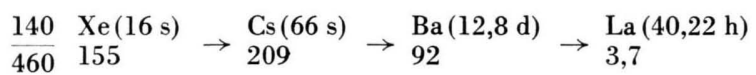

$$
\begin{aligned}
& \begin{array}{l}
141 \mathrm{Xe}(1,7 \mathrm{~s}) \\
430 \mathrm{Cs}(9 \mathrm{~s})
\end{array} \rightarrow \begin{array}{l}
\mathrm{Ba}(18 \mathrm{~m}) \\
142
\end{array} \rightarrow \begin{array}{l}
\mathrm{La}(3,8 \mathrm{~h}) \\
23,6
\end{array} \rightarrow \begin{array}{l}
\mathrm{Ce}(33,1 \mathrm{~d}) \\
0,047
\end{array}
\end{aligned}
$$

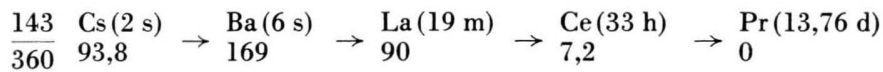

$$
\begin{aligned}
& \begin{array}{l}
144 \mathrm{Cs}(1,5 \mathrm{~s}) \\
330
\end{array} \rightarrow \begin{array}{l}
\mathrm{Ba}(3,5 \mathrm{~s}) \\
142
\end{array} \rightarrow \begin{array}{l}
\mathrm{La}(15 \mathrm{~s}) \\
116
\end{array} \rightarrow \begin{array}{l}
\mathrm{Ce}(285 \mathrm{~d}) \\
24,8
\end{array} \rightarrow \begin{array}{l}
\operatorname{Pr}(17,27 \mathrm{~m}) \\
0,036
\end{array}
\end{aligned}
$$

Tab. 2. Spaltausbeute, Halbwertszeit und Umwandlungsreihe verschiedener Massenzahlen, die bei der Spaltung von Uran-238 mit 14 MeT-Neutronen entstehen ${ }^{5}$. - Neben dem Elementsymbol-steht in Klammern die Halbwertszeit, darunter die Spaltausbeute.

aktiven Isotope. Die zum $\mathrm{Zr}-\mathrm{Nb}-95$ führende Zerfallsreihe (eine Auswahl der von Dolan ${ }^{5}$ zusammengestellten Liste ist in Tab. 2 enthalten) verläuft über die früh kondensierenden Elemente Sr und Y. Daher wird Zr-Nb-95 bei Atombomben aller Kaliber (bei großen sogar noch bevorzugt wegen des späten „absoluten“ Zeitbeginnes der Kondensation, so daß $\mathrm{Kr}$ und $\mathrm{Rb}$ zu Sr bzw. Y zerfallen sind) ein wesentlicher Bestandteil der zuerst gebildeten Partikel sein. In ihnen sind etwa gleichviel Atome vom kurzlebigen Mo-99 zu erwarten. Dann folgen mit Anteilen, die im Vergleich zur Spaltausbeute vermindert sind, Ce-141, Ce-Pr-144, Ba-La-140, I-131, Pr-143, NdPm-147 und andere Isotope geringer Spaltanteile. Für Sr-89, Sr-90 und Cs-137, die überwiegend über relativ langlebige (im Vergleich zur Lebensdauer des Feuerballs) Edelgase und andere flüchtige Elemente entstehen, ist starke Abreicherung zu erwarten.

Im Verlauf der Feuerballentwicklung beginnt die Kondensation des Lanthan zusammen mit der von $\mathrm{Sr}, \mathrm{Y}$ (und Zr) zum relativen Zeitpunkt $t / t_{2} \sim 24$ 
(Abb. 3). Da im weiteren Verlauf die Anlagerungswahrscheinlichkeit an ein bei Beginn der Abscheidung vorhandenes oder gebildetes Teilchen rasch abnimmt, wird die Hauptmasse des Lanthan in einer kurzen Zeitspanne nach dem Kondensationsbeginn abgeschieden und später absorbierte Anteile können in erster Näherung vernachlässigt werden. Wäre Lanthan in den zu Ce-141 und Ce-144 führenden Zerfallsreihen (Tab. 2) das einzige kondensierende Element, so wäre das beobachtete Ce-144/Ce-141durch das La-144/La-141-Verhältnis im Zeitpunkt der Anlagerung bestimmt. Da dieses Verhältnis zeitveränderlich ist (Abb. $4 \mathrm{~b}$ ) und da andererseits der „absolute“ Zeitpunkt des Kondensationsbeginns je nach dem Bombenkaliber zwischen etwa 1 und $130 \mathrm{sec}$ [Gl. (2) ] liegt, ergäbe sich eine einfache Möglichkeit der Abschätzung der Explosionsstärke der Bombe. Wegen der später erfolgenden aber noch merklichen Anlagerung von Ba und der nach Abb. 3 im gleichen $\mathrm{Ma} \beta$ zu erwartenden Abscheidung von Ce werden außer La-141 und La-144 auch die Isotope Ba-141, Ba-144, Ce-141 und Ce-144 noch zu dem schließlich gemessenen Ce-141 und Ce-144 beitragen. Dadurch werden die Verhältnisse komplizierter. Bei dem untersuchten Teilchen wurde das Verhältnis von Ba-140/Zr-95 gemessen. Daher ist der Diskriminierungsfaktor für Ba-141, Ba-144, Ce-141 und Ce-144 bekannt und aus den beobachteten Zr-95-

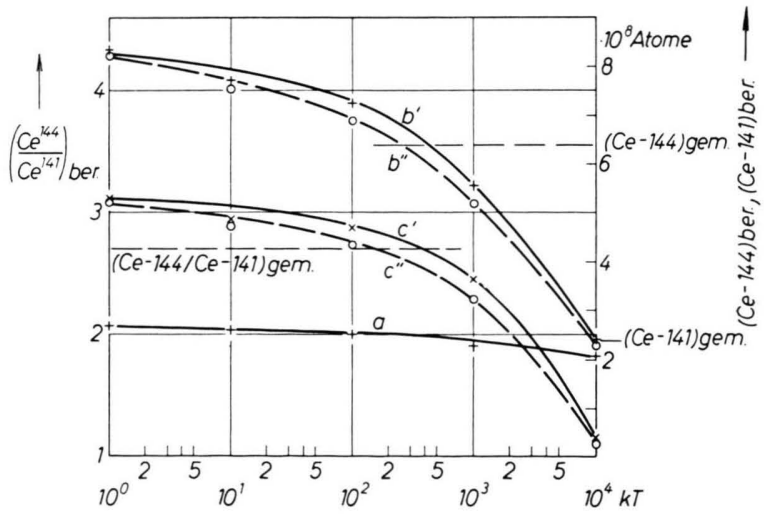

Ab. 5. Aus dem Zirkon-95- und Barium-140-Gehalt des untersuchten Teilchens errechneter Verlauf der Cer-141- (a) und 144- (b) Anteile und des Cer-144/Cer-141-Verhältnisses (c) in Abhängigkeit von der Explosionsenergie einer Plutonium239-Luftexplosion.

16 Dabei wurde angenommen, daß in der Partikel entstehendes Cer nicht wieder abgedampft ist.

17 Mit U-233 als Energielieferant der Explosion ist das Massenausbeuteverhältnis 144/141 noch kleiner als bei U-235 oder U.238 zu erwarten, so daß sich das beobachtete und Ba-140-Intensitäten lassen sich die zu erwartenden Ce-141- und Ce-144-Anteile bestimmen. Die Rechnung wurde für das oben benutzte Modell der Luftexplosion einer Pu-239-Spaltbombe durchgeführt. Das Ergebnis ist in Abb. 5 dargestellt. Mit den gemachten Annahmen läßt sich der gemessene Ce-141- und Ce-144-Gehalt und das Ce-144/Ce-141Verhältnis (zwei unabhängige Größen) erklären, wenn das Teilchen von einer Explosion mit einer Energie von etwa $400 \mathrm{kT}$ TNT-Äquivalent stammt. Zur Rechnung wurden die Entwicklungskurven der verschiedenen Isotope benutzt (Abb. $4 \mathrm{~b})$, die sich aus den Spaltausbeuten und den Halbwertszeiten der beteiligten Partner ergeben. Da aber Lanthan früher als Cer kondensiert, wird nach seiner $\mathrm{Ab}$ scheidung in der Gasphase praktisch kein Cer mehr nachgebildet ${ }^{16}$. Bei Berücksichtigung dieses Effektes wird die Ce-144-Kurve der Abb. 5 im Bereich von $10^{2}-10^{3} \mathrm{kT}$ abgeflacht (gestrichelte Kurven) und die gemessenen Werte führen zu einer Explosionsstärke von $100-300 \mathrm{kT}$ TNT. Zur Abschätzung der Einflüsse wurden die Berechnungen der Cer-Anteile aus dem Zr-95- und Ba-140-Gehalt auch mit dem Millerschen ${ }^{6}$ Modell einer Oberflächenexplosion und für den Fall durchgeführt, daß Pu-239 als Zünder einer $\mathrm{H}$-Bombe nur 1/10 der Explosionsenergie liefert. In beiden Fällen ergeben sich nur geringe Abweichungen. Solange Spaltung von $\mathrm{Pu}-239$ angenommen werden kann, ergibt sich die Explosionsstärke zu 100 bis 300 kT TNT-Äquivalent. Da Pu239 nicht das einzige für Atombomben verwendbare Material ist, wurde die Cer-Berechnung für U-238 und U-235 (nur für diese Elemente standen Spaltausbeutewerte zur Verfügung) wiederholt. Bei diesen Isotopen ist das Massenausbeuteverhältnis 144/141 etwa 20\% kleiner als bei Pu-239. Dies führt dazu, daß das maximal erreichbare Verhältnis für Ce-144/Ce-141 unter 2,7 sinkt, so daß die Entstehung des Teilchens bei einer derartigen Explosion wenig wahrscheinlich wird. Wegen der Unsicherheiten der Messung und der sonst verwendeten Angaben läßt sich aber eine kleine (taktische) Bombe nicht mit Sicherheit ausschließen. Insbesondere könnte das beobachtete Cer-Verhältnis mit einer passenden Mischung von $\mathrm{Pu}-239$ und anderem Spalt-

Ce-144/Ce-141-Verhältnis noch schlechter erklären ließe. Die Abschätzung der Mindestenergie der Explosion aus dem Fehlen der Ru-103-Linie im $\gamma$-Spektrum ist in diesem Falle, da die Spaltausbeute der Masse 103 noch ungünstiger sein wird als bei U-235, nicht anwendbar. 
material bei energiearmen Explosionen erreicht werden. In diesem Fall liegt aber der Beginn der Spaltproduktkondensation bei so kleinen Zeiten, daß in der zum Ru-103 führenden Zerfallsreihe Zr-103 (1,5 sec) und Nb-103 (4 sec) noch nicht abgeklungen sind. Sie müßten im Teilchen eingebaut werden und zu einer Ru-Rh-103-Aktivität, bezogen auf die von $\mathrm{Zr}-\mathrm{Nb}-95$, von etwa $38 \%$ bei einer $1 \mathrm{kT}$-Bombe bzw. $20 \%$ bei $10 \mathrm{kT}$ bzw. $5 \%$ bei $100 \mathrm{kT}$ führen mit Pu-239 und U-238 als Ausgangsmaterial. Bei U-235 wären die Anteile nur 1/3 der oben angegebenen Werte. Da bei der $\gamma$-Analyse des Teilchens der Anteil der Ru-Rh-103-Aktivität 5\% der Zr-Nb-95-Aktivität nicht überstieg, spricht das Ergebnis der Analyse für eine Bombe mit mehr als $100 \mathrm{kT}$ TNT-Energie und $\mathrm{Pu}-239$ als Spaltmaterial ${ }^{17}$.

Aus der durch das Feuerballmodell gegebenen Spaltproduktdichte zum Zeitpunkt der Kondensation und der im Teilchen vorhandenen Zr-95-Menge ergibt sich das bei der Abscheidung erfaßte Volumen. Für eine $200 \mathrm{kT}$ Plutoniumspaltbombe beträgt es bei einer Luftexplosion etwa $0,60 \mathrm{~cm}^{3}$, bei einer Bodenexplosion etwa $0,22 \mathrm{~cm}^{3}$. Mit der Annahme, daß auch der festgestellte Eisen- und Aluminiumgehalt der Partikel aus der Gasphase ausgeschieden wurde, errechnet sich der Gehalt dieser Elemente beim Kondensationsbeginn zu etwa $3,3 \cdot 10^{13}$ bzw. $8,8 \cdot 10^{13}$ Atome $/ \mathrm{cm}^{3}$. Die mit dieser Dichte ermittelten Partialdrucke erreichen den Sättigungsdampfdruck der Oxyde bei $t / t_{2} \sim 22$ für Eisen und $t / t_{2} \sim 16$ für Aluminium. Der Kondensationspunkt für Eisenoxyd liegt nahe bei dem der zum Zr-95 führenden Isotope, so daß die Annahme gleicher Kondensationsvolumen gerechtfertigt erscheint. Die Abscheidung von Aluminium erfolgt dagegen so viel früher, daß die Zulässigkeit der Dichteabschätzung zweifelhaft

18 Nach der durchgeführten Analyse wurde das Teilchen bei einer Explosion von 100 bis 300 kT TNT-Äquivalent gebildet. Für eine reine Spaltbombe ist dazu eine Plutoniummenge notwendig, die wesentlich größer ist als die kriti- wird. Durch den Zeitpunkt der Kondensation ist das dazu gehörende Feuerballvolumen festgelegt [Gl. (5) bzw. (8) ] und mit der oben bestimmten Dichte errechnet sich das Gewicht des im Feuerball vorhandenen Eisen zu etwa $2200 \mathrm{~kg}$ (das des Aluminiums zu etwa $580 \mathrm{~kg}$ ) für beide Modelle. Die ermittelten Eisen- und Aluminiumgewichte sind sehr hoch. Wenn die Energie der Explosion nur zu einem Teil durch die Spaltung von Pu-239 gedeckt wird ${ }^{18}$, sind wegen der verminderten Spaltproduktdichte größere Kondensationsvolumen nötig, um den Zr-95-Gehalt des heißen Teilchens zu erklären. Dann sinkt die abgeschätzte Fe- bzw. Al-Dampfdichte und die in den Feuerball einbezogene Menge vermindert sich auf etwa den Bruchteil der durch das Verhältnis des Anteils der Spaltenergie zur Gesamtenergie der Explosion gegeben ist.

Wegen der unvollkommenen Kenntnis der bei einer Atombombenexplosion ablaufenden Vorgänge mußten bei den durchgeführten Abschätzungen starke Vereinfachungen gemacht werden. Das Bild der Entstehung der "heißen“ Teilchen ist daher nur eine grobe Näherung. Das Ergebnis der experimentellen Untersuchungen an zahlreichen Einzelteilchen des weltweiten Fallout zeigt, daß die Vorstellung über die stufenweise Kondensation der verschiedenen Elemente entsprechend der Flüchtigkeit der Oxyde sinnvoll ist. $\mathrm{Ob}$ auch der weitergehende Schluß auf die Stärke der erzeugenden Explosion und die in den Feuerball einbezogene Materialmenge vernünftig ist, kann wegen des Fehlens von Unterlagen nicht entschieden werden.

Die Untersuchungen wurden mit Unterstützung der Schutzkommission beim Bundesministerium des Innern durchgeführt.

sche Masse. Die Verwendung von soviel Plutonium kann nicht ausgeschlossen werden. Andererseits scheint auch möglich, daß bei dem Versuch eine kleine „saubere“ Wasserstoffbombe mit einem Plutoniumzünder erprobt wurde. 